\title{
Bandwidth Extension of High Compliance Current Mirror by Using Compensation Methods
}

\author{
Maneesha Gupta, Urvashi Singh, and Richa Srivastava \\ Netaji Subhash Institute of Technology, Dwarka Sector 3, New Delhi 110078, India \\ Correspondence should be addressed to Urvashi Singh; urvashi.singh27@gmail.com
}

Received 19 October 2013; Revised 6 December 2013; Accepted 6 December 2013; Published 21 January 2014

Academic Editor: Gerard Ghibaudo

Copyright ( 2014 Maneesha Gupta et al. This is an open access article distributed under the Creative Commons Attribution License, which permits unrestricted use, distribution, and reproduction in any medium, provided the original work is properly cited.

\begin{abstract}
Due to the huge demand of high-speed analog integrated circuits, it is essential to develop a wideband low input impedance current mirror that can be operated at low power supply. In this paper, a novel wideband low voltage high compliance current mirror using low voltage cascode current mirror (LVCCM) as a basic building block is proposed. The resistive compensation and inductive peaking methods have been used to extend the bandwidth of the conventional current mirror. By replacing conventional LVCCM in a high compliance current mirror with the compensated LVCCM, the bandwidth extension ratio of 3.4 has been achieved with no additional DC power dissipation and without affecting its other performances. The circuits are designed in TSMC $0.18 \mu \mathrm{m}$ CMOS technology on Spectre simulator of Cadence.
\end{abstract}

\section{Introduction}

In today's electronics world, the demand of high-speed analog devices has increased tremendously due to the explosive growth of communication systems $[1,2]$. Moreover, the importance of low power and low voltage analog and mixedsignal circuits is increasing with the need of portable electronics devices. Since the current-mode devices can operate at low voltage and has higher bandwidth than that of voltagemode devices, these devices are becoming the first preference of designers for signal processing applications $[3,4]$.

Current mirror is one of the most widely used currentmode circuit in analog integrated circuits such as operational amplifiers, current-mode analog-to-digital and digital-toanalog data converters, artificial neural networks, current sensing circuits, current-mode filters, current conveyors, and translinear loops [5-10]. Current mirrors are basically used for current amplification, level shifting, biasing, and loading in a circuit. A current mirror should have low input impedance and high output impedance for proper functionality. Other desirable features of a current mirror include wide input and output current swings, high linearity, accurate current copy, and low standby power dissipation.
But a conventional current mirror (i.e., a current mirror which consists of two MOS transistors) has low output impedance and high current transfer error. Cascoding of transistors improves the output impedance and accuracy, but it also increases the power supply requirement and decreases input/output compliances. Several low voltage current mirrors are reported in [11-14]. A low voltage cascode current mirror (LVCCM) is one of the efficient and simple current mirrors. It provides low input impedance and high output impedance with reduced power supply requirement (shown in Figure 1). It has been used in many analog and mixed signal circuits [15-18].

The input impedance and input voltage can be lowered to the minimum value by applying input current at the output node of the flipped voltage follower (FVF) of LVCCM as shown in Figure 2.

In this paper, conventional LVCCM (Figure 2) is analyzed at high frequency and its bandwidth is calculated. It is found that the bandwidth of LVCCM is not large enough for high-speed applications. The bandwidth of a circuit can be enhanced by using resistive compensation technique [20, 21], inductive peaking technique $[22,23]$, negative capacitance compensation [24], feedforward compensation [25], and so 


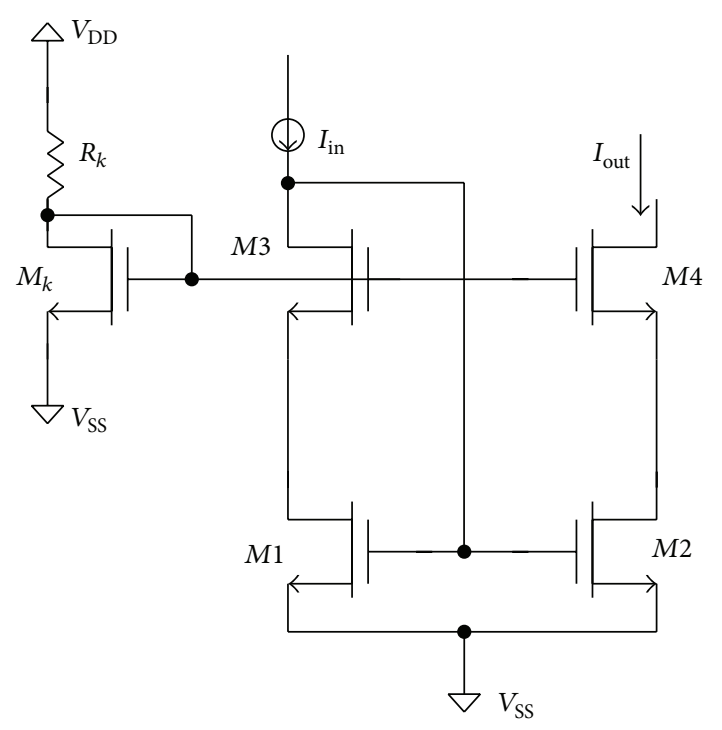

Figure 1: Conventional low voltage cascode current mirror (LVCCM), when input current is fed at the drain terminal of transistor M3.

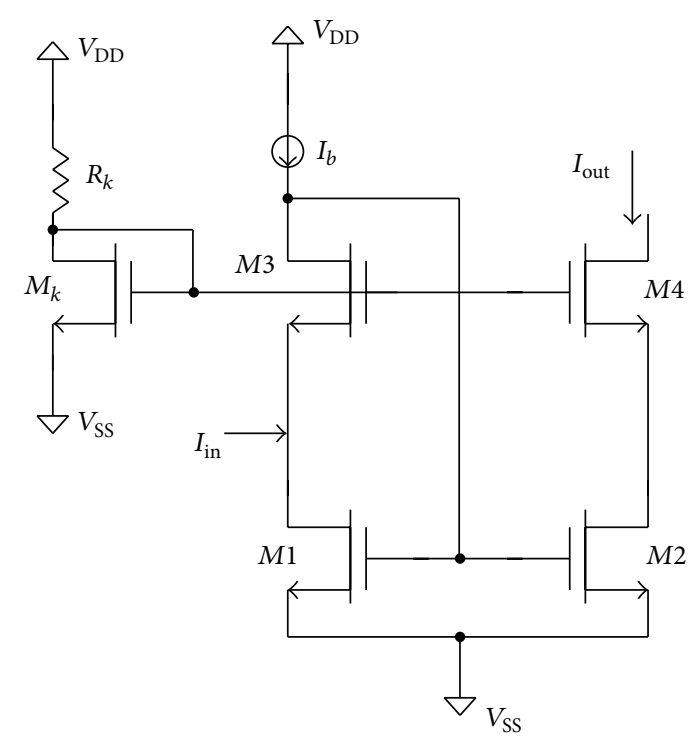

FIgure 2: Conventional LVCCM, when input current is fed at the drain terminal of $M 1$.

forth. The bandwidth of LVCCM has been enhanced by Gupta et al. in [26] by using resistive compensation method. In this work, both resistive and inductive compensation techniques are used to extend the bandwidth of conventional LVCCM, and the maximum obtained bandwidth extension ratio (BWER) is 3.82 by using $20 \mathrm{nH}$ inductance between gate terminals of transistors $M 1$ and $M 2$ in LVCCM (Figure 2).

The FVF based LVCCM has an offset which can be reduced by providing a negative feedback in the circuit. Javad Azhari et al. [19] have developed a current mirror which has reduced offset effect at the output. In this paper the $-3 \mathrm{~dB}$ frequency of the high compliance current mirror [19] is enhanced by using compensated high frequency LVCCM.

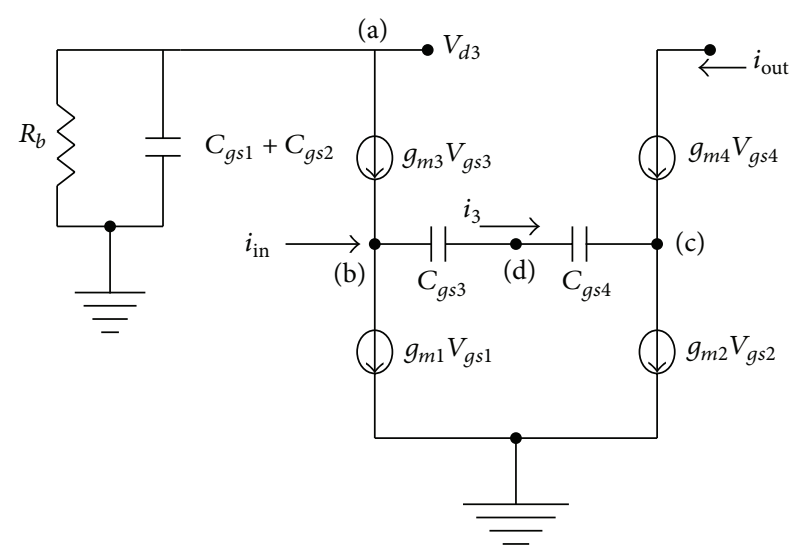

FIGURE 3: Small signal model of LVCCM (shown in Figure 2).

The paper is organized in 6 sections. Section 2 includes small signal analysis of conventional LVCCM. The application of compensation methods in conventional LVCCM is explained in Section 3. The wideband high compliance current mirror is proposed in Section 4. Simulation results are given in Section 5 and then conclusions are drawn in the last section.

\section{Small Signal Analysis of Conventional LVCCM}

The small signal model of conventional LVCCM is shown in Figure 3 . The channel-length modulation effect of transistors has been ignored. It has also been assumed that the substrate of each transistor is connected to the source terminal of transistor, so that there is no body effect and simulations are also performed on the same basis.

In analytical derivations $C_{g s i}$ and $g_{m i}$ are the gate to source capacitance and transconductance of transistor $M i$, respectively (where $i=1$ to 4 ).

Applying KCL at nodes (b) and (c) in Figure 3, we get

$$
\begin{gathered}
i_{\text {in }}+g_{m 3} v_{g s 3}=g_{m 1} v_{g s 1}+i_{1}, \\
g_{m 4} v_{g s 4}+i_{1}=g_{m 2} v_{g s 2} .
\end{gathered}
$$

Substituting $i_{1}$ from (1) into (2),

$$
i_{\text {in }}+g_{m 3} v_{g s 3}=g_{m 1} v_{g s 1}+g_{m 2} v_{g s 2}-g_{m 4} v_{g s 4} .
$$

Neglecting channel-length modulation effect, the output current $i_{\text {out }}$ is

$$
i_{\text {out }}=g_{m 4} v_{g s 4} .
$$

Using (4) in (3), we obtain

$$
i_{\text {in }}+g_{m 3} v_{g s 3}=g_{m 1} v_{g s 1}+g_{m 2} v_{g s 2}-i_{\text {out }} .
$$

Also, on performing nodal analysis at node (c), we get

$$
\left(v_{s 4}-v_{g 4}\right) s C_{g s 4}+g_{m 2} v_{g s 2}=i_{\text {out }} .
$$


Then $v_{g s 2}$ can be obtained as

$$
v_{g s 2}=\left(\frac{i_{\text {out }}}{g_{m 2}}\right)+\left(\frac{s C_{g s 4} v_{g s 4}}{g_{m 2}}\right) .
$$

Substituting $v_{g s 4}$ from (4) into (7),

$$
v_{g s 2}=\left(\frac{i_{\text {out }}}{g_{m 2}}\right)\left(1+\frac{s C_{g s 4}}{g_{m 4}}\right) .
$$

At node (d), we have

$$
\begin{gathered}
i_{1}=-s C_{g s 3} v_{g s 3}=s C_{g s 4} v_{g s 4}, \\
v_{g s 3}=-v_{g s 4}\left(\frac{C_{g s 4}}{C_{g s 3}}\right)=-i_{\text {out }}\left(\frac{C_{g s 4}}{g_{m 4} C_{g s 3}}\right) .
\end{gathered}
$$

Substituting $v_{g s 3}$ and $v_{g s 2}$ from (10) and (8), respectively, into (5),

$$
\begin{aligned}
i_{\text {in }} & -i_{\text {out }}\left(\frac{g_{m 3} C_{g s 4}}{g_{m 4} C_{g s 3}}\right) \\
& =i_{\text {out }}\left\{\frac{\left(g_{m 1}+g_{m 2}\right)\left(g_{m 4}+s C_{g s 4}\right)}{g_{m 2} g_{m 4}}-1\right\} .
\end{aligned}
$$

Simplifying (11) to obtain small signal current gain $A_{i}(s)$,

$$
\begin{aligned}
A_{i}(s)= & \left(\frac{i_{\text {out }}}{i_{\text {in }}}\right) \\
= & g_{m 2} g_{m 4} C_{g s 3} \\
& \times\left\{s C_{g s 3} C_{g s 4}\left(g_{m 1}+g_{m 2}\right)+g_{m 1} g_{m 4} C_{g s 3}\right. \\
& \left.\quad+g_{m 2} g_{m 3} C_{g s 4}\right\}^{-1} .
\end{aligned}
$$

Let us assume $g_{m 1}=g_{m 2}$ and $C_{g s 3}=C_{g s 4}=C_{g s}$; then,

$$
A_{i}(s)=\frac{g_{m 4}}{2 s C_{g s}+g_{m 4}+g_{m 3}} .
$$

If we choose $g_{m 3}=g_{m 4}=g_{m}$,

$$
A_{i}(s)=\left(\frac{g_{m}}{2 C_{g s}}\right)\left(\frac{1}{s+g_{m} / C_{g s}}\right) .
$$

The bandwidth of conventional LVCCM is given by

$$
\omega_{0}=\left(\frac{g_{m}}{C_{g s}}\right)
$$

\section{High Frequency LVCCM}

The circuit of the modified LVCCM is shown in Figure 4. An impedance $Z$ is inserted between the gates of transistors $M 1$ and $M 2$ [20-23]. The impedance $Z$ can be a resistance $(R)$ or inductance $(L)$.

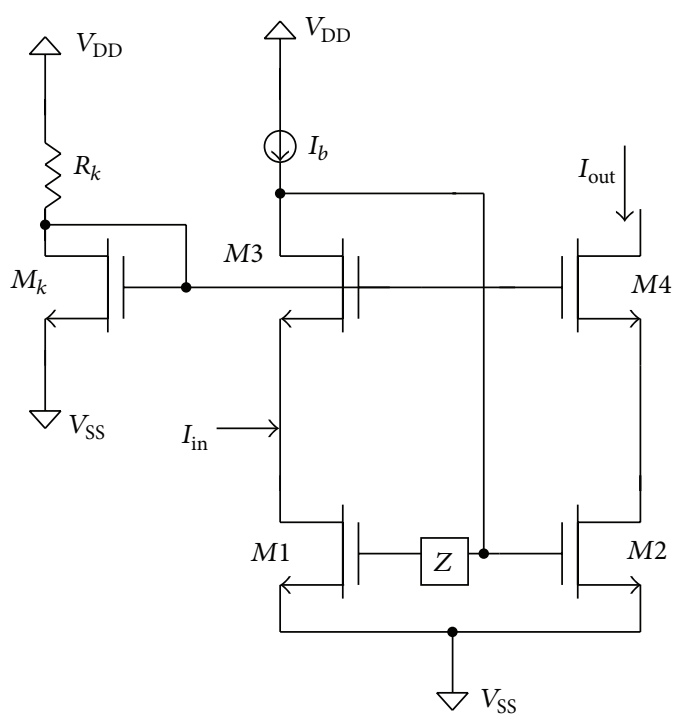

FIGURE 4: Compensated LVCCM.

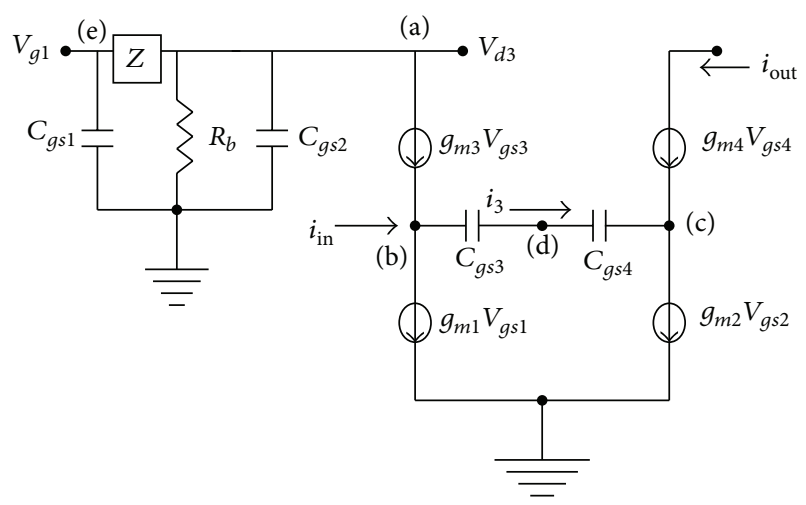

FIGURE 5: Small signal model of the compensated LVCCM.

3.1. Small Signal Analysis. The small signal model of the modified LVCCM is shown in Figure 5. The analysis has been done on the basis of same assumptions as taken in Section 2.

On applying KCL at nodes (b) and (c) in Figure 5, we get

$$
i_{\text {in }}=g_{m 1} v_{g s 1}+g_{m 2} v_{g s 2}-i_{\text {out }}-g_{m 3} v_{g s 3},
$$

where $v_{g s 1}$ is given by

$$
v_{g s 1}=v_{g s 2}\left(\frac{1}{1+s C_{g s 1} Z}\right) .
$$

From (7), $v_{g s 2}=\left(i_{\text {out }} / g_{m 2}\right)\left(1+s C_{g s 4} / g_{m 4}\right)$; then (17), can be modified into

$$
v_{g s 1}=\left(\frac{i_{\text {out }}}{g_{m 2}\left(1+s C_{g s 1} Z\right)}\right)\left(1+\frac{s C_{g s 4}}{g_{m 4}}\right) .
$$


Using (7), (10) and (18), and performing some simplifications on (16) to obtain current transfer function of the compensated LVCCM. The obtained $A_{i}(s)$ is

$$
\begin{aligned}
A_{i}(s)= & \left(\frac{i_{\text {out }}}{i_{\text {in }}}\right) \\
= & g_{m 2} g_{m 4} C_{g s 3}\left(1+s C_{g s 1} Z\right) \\
& \times\left\{s^{2} g_{m 2} C_{g s 1} C_{g s 3} C_{g s 4} Z+s C_{g s 4}\right. \\
& \times\left(g_{m 1} C_{g s 3}+g_{m 2} C_{g s 3}+g_{m 2} g_{m 3} C_{g s 1} Z\right) \\
& \left.+g_{m 1} g_{m 4} C_{g s 3}+g_{m 2} g_{m 3} C_{g s 4}\right\}^{-1} .
\end{aligned}
$$

3.1.1. Bandwidth Extension Using Resistive Compensation Method. On taking $Z=R$ (i.e., resistive compensation method), then the current transfer function becomes

$$
\begin{aligned}
A_{i}(s)= & \left(\frac{i_{\text {out }}}{i_{\text {in }}}\right) \\
= & g_{m 2} g_{m 4} C_{g s 3}\left(1+s C_{g s 1} R\right) \\
& \times\left\{s^{2} g_{m 2} C_{g s 1} C_{g s 3} C_{g s 4} R+s C_{g s 4}\right. \\
& \times\left(g_{m 1} C_{g s 3}+g_{m 2} C_{g s 3}+g_{m 2} g_{m 3} C_{g s 1} R\right) \\
& \left.+g_{m 1} g_{m 4} C_{g s 3}+g_{m 2} g_{m 3} C_{g s 4}\right\}^{-1} .
\end{aligned}
$$

Again, if we assume $g_{m 1}=g_{m 2}$ and $C_{g s 3}=C_{g s 4}=C_{g s}$, then (20) gets transformed into

$$
\begin{aligned}
& A_{i}(s) \\
& \quad=\frac{g_{m 4}\left(1+s C_{g s 1} R\right)}{\left\{s^{2} C_{g s 1} C_{g s} R+s\left(2 C_{g s}+g_{m 3} C_{g s 1} R\right)+g_{m 4}+g_{m 3}\right\}} .
\end{aligned}
$$

Let us suppose $g_{m 3}=g_{m 4}=g_{m}$; then, $A_{i}(s)$ is

$$
A_{i}(s)=\frac{g_{m}\left(1+s C_{g s} R\right)}{s^{2} C_{g s}^{2} R+s\left(2 C_{g s}+g_{m} C_{g s} R\right)+2 g_{m}} .
$$

Equation (22) can be expressed as

$$
\begin{aligned}
A_{i} & (s) \\
\quad= & \left(\frac{g_{m}}{C_{g s}}\right) \frac{\left(s+1 / C_{g s} R\right)}{\left\{s^{2}+s\left(2 / C_{g s} R+g_{m} / C_{g s}\right)+\left(2 g_{m} / C_{g s}^{2} R\right)\right\}} .
\end{aligned}
$$

The transfer function of modified LVCCM is of second order with one zero and two poles. The zero and poles of the modified LVCCM are

$$
\begin{aligned}
& Z_{1}=-\left(\frac{1}{C_{g s} R}\right), \\
& P_{1}=-\left(\frac{g_{m}}{C_{g s}}\right), \\
& P_{2}=-\left(\frac{2}{C_{g s} R}\right) .
\end{aligned}
$$

At $R=1 / g_{m}$, one pole-zero pair $\left(P_{1}-Z_{1}\right)$ gets cancelled and the current transfer function (see (23)) will transform into single pole low pass system and has bandwidth $\omega_{0 R}=$ $\left(2 g_{m} / C_{g s}\right)$ which is double of the conventional LVCCM (see (15)).

3.1.2. Bandwidth Extension Using Inductive Peaking Method. On taking $Z=s L$ (i.e., inductive peaking method), the current transfer function of the modified LVCCM is given by

$$
\begin{aligned}
A_{i}(s)= & \left(\frac{i_{\text {out }}}{i_{\text {in }}}\right) \\
= & g_{m 2} g_{m 4} C_{g s 3}\left(1+s^{2} C_{g s 1} L\right) \\
& \times\left\{s^{3} g_{m 2} C_{g s 1} C_{g s 3} C_{g s 4} L+s^{2} g_{m 2} g_{m 3} C_{g s 1} C_{g s 4} L\right. \\
& \quad+s C_{g s 4}\left(g_{m 1} C_{g s 3}+g_{m 2} C_{g s 3}\right) \\
& \left.\quad+g_{m 1} g_{m 4} C_{g s 3}+g_{m 2} g_{m 3} C_{g s 4}\right\}^{-1} .
\end{aligned}
$$

The effect of using an inductor on small signal current gain can be observed from (25); it adds a zero and a pole to the transfer function which allows more control over the frequency response of the LVCCM [27].

By definition [28], at $\omega=\omega_{0}$,

$$
\left|A_{i}\left(\omega_{0}\right)\right|^{2}=0.5\left|A_{i}(0)\right|^{2},
$$

where $\left|A_{i}(0)\right|^{2}=1$ of a current mirror.

From (25) and (26), the bandwidth of the proposed LVCCM is given by

$$
\omega_{0} \cong \sqrt{\left(\frac{2}{C_{g s} L}\right)-\left(\frac{g_{m}}{C_{g s}}\right)^{2}} .
$$

It is obvious form (27) that the $-3 \mathrm{~dB}$ frequency of the inductively peaked LVCCM depends on the value of inductor.

At $L=\left(C_{g s} / 5 g_{m}^{2}\right)$, the bandwidth of the wideband current mirror becomes

$$
\omega_{0 L} \cong\left(\frac{3 g_{m}}{C_{g s}}\right) .
$$

The bandwidth of the inductively peaked LVCCM which is thrice of the conventional LVCCM for the chosen value of inductor as shown in (28). 


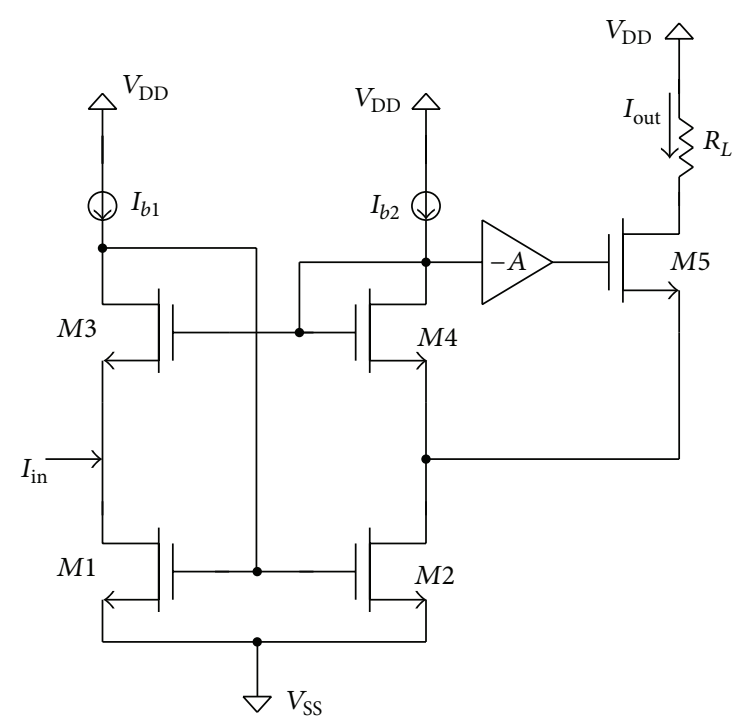

FIGURE 6: High compliance current mirror [19].

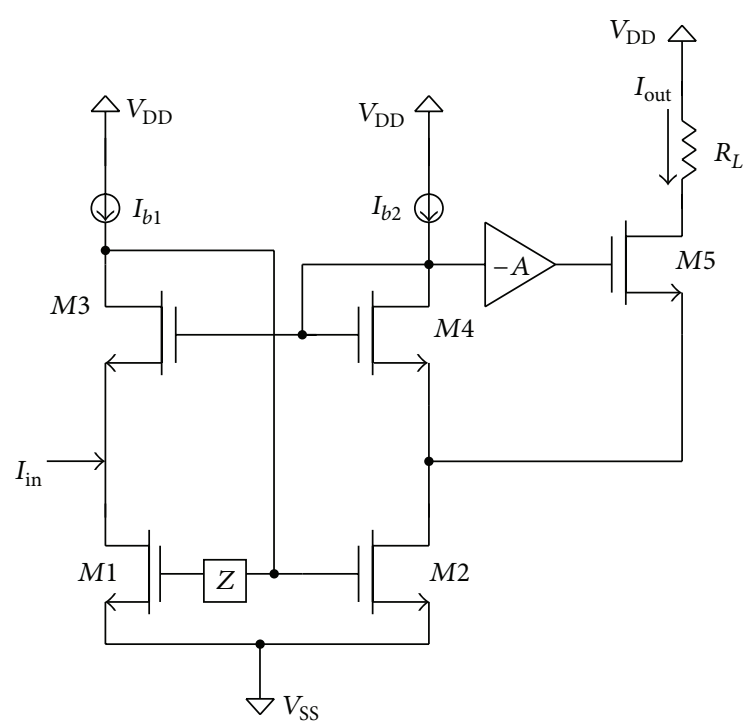

Figure 7: Proposed wideband high compliance current mirror.

TABLE 1: Circuit parameters.

\begin{tabular}{lc}
\hline Parameters & Value \\
\hline Power supply $\left(V_{\mathrm{DD}}\right)$ & $1.5 \mathrm{~V}$ \\
Aspect ratio $(M 1=M 2)$ & $(5.4 / 0.18) \mu \mathrm{m}$ \\
Aspect ratio $(M 3=M 4)$ & $(3.6 / 0.18) \mu \mathrm{m}$ \\
Aspect ratio $(M 5)$ & $(18 / 0.18) \mu \mathrm{m}$ \\
Bias current $\left(I_{b}\right)$ & $100 \mu \mathrm{A}$ \\
Bias resistance $\left(R_{k}\right)$ & $3.3 \mathrm{M} \Omega$ \\
\hline
\end{tabular}

\section{Proposed High Frequency Current Mirror}

Due to the low voltage requirement, low input impedance, and high accuracy, LVCCM is used as a basic building block in many analog and mixed signal systems. Javad Azhari et

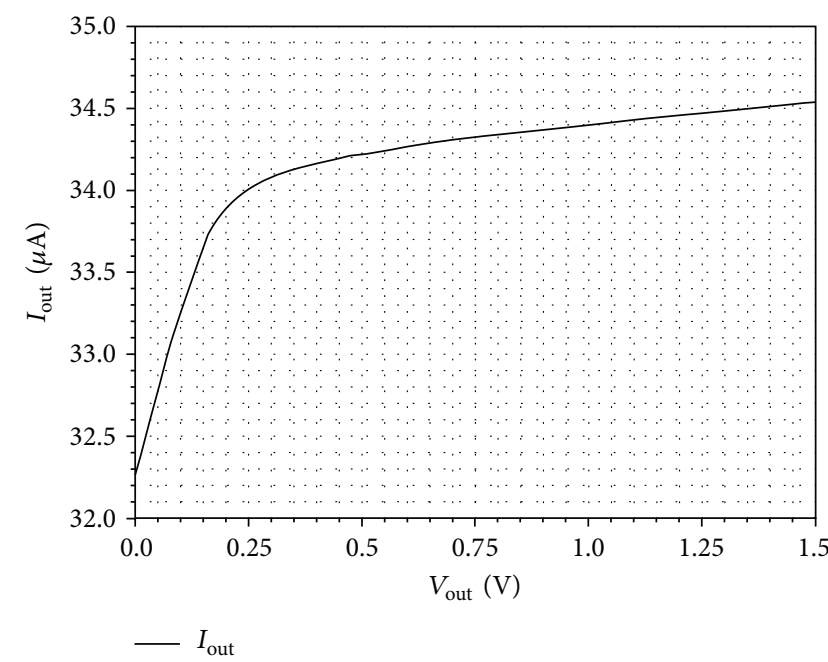

FIGURE 8: Variation of output current with respect to output voltage of LVCCM.

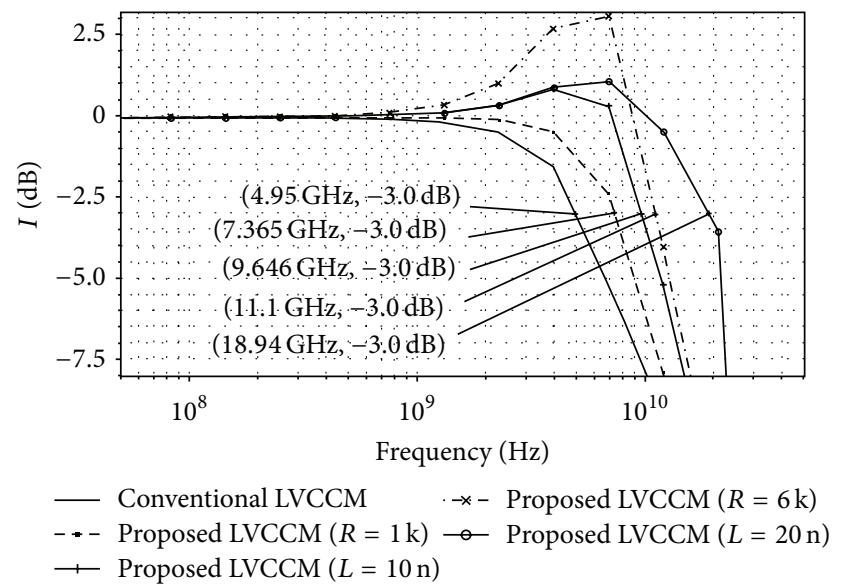

FIGURE 9: Frequency responses of designed LVCCM.

al. [19] have implemented a high compliance current mirror (shown in Figure 6) with a feedback mechanism to eliminate the offset current produced in LVCCM (when input current is fed at the drain terminal of transistor $M 1$; Figure 2).

The performance of current mirror has been improved in [19] by providing a feedback network, but its bandwidth is degraded. In this paper, we have modified the current mirror [19] by replacing the conventional LVCCM with the proposed wideband LVCCM to obtain a wideband high performance current mirror. The proposed current mirror is shown in Figure 7.

The bandwidth of the current mirror is enhanced with no additional DC power dissipation and without affecting the DC characteristics such as linearity.

\section{Simulation Results and Discussion}

The circuits are designed in TSMC $0.18 \mu \mathrm{m}$ CMOS technology and simulated with Spectre simulator of Cadence. The circuit parameters of LVCCM are given in Table 1. 
TABLE 2: Simulated circuit parameters.

\begin{tabular}{lcccc}
\hline Parameters & $M 1$ & $M 2$ & $M 3$ & $M 4$ \\
\hline $\begin{array}{l}\text { Transconductance } \\
\left(g_{m} \times 10^{-6}\right) \mathrm{A} / \mathrm{V}\end{array}$ & 526.261 & 585.829 & 411.018 & 399.300 \\
$\begin{array}{l}\text { Gate to source capacitance } \\
\left(C_{g s} \times 10^{-15}\right) \mathrm{F}\end{array}$ & 11.010 & 15.670 & 7.842 & 7.131 \\
$\begin{array}{l}\text { Overdrive voltage } \\
\left(V_{g s}-V_{\text {th }}\right) \times 10^{-3} \mathrm{~V}\end{array}$ & 0.522 & 0.519 & 84.454 & 89.751 \\
\hline
\end{tabular}

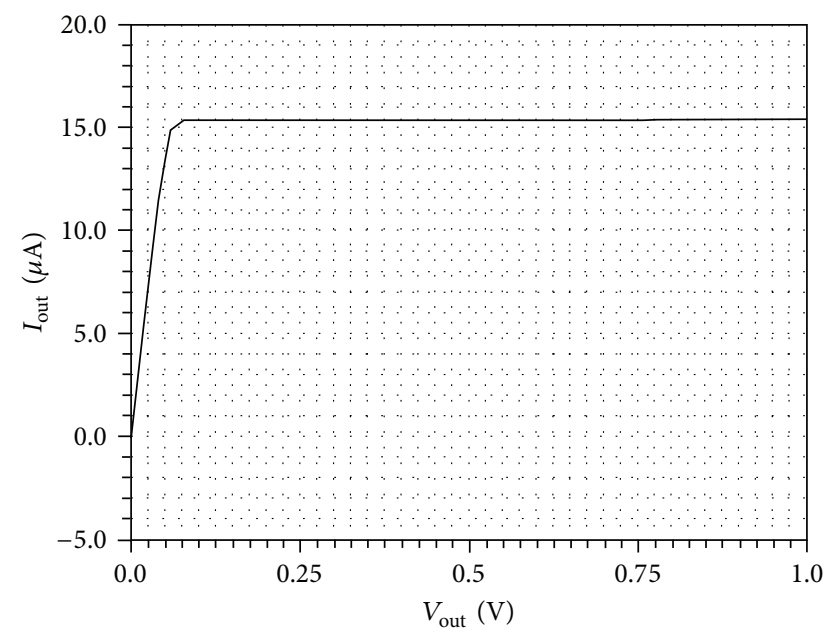

FIGURE 10: Variation of output current with respect to output voltage of the proposed current mirror.

The simulated values of transconductances, gate to source capacitances, and overdrive voltages of transistors $M 1, M 2$, M3, and M4 are given in Table 2.

The $-3 \mathrm{~dB}$ frequencies of designed current mirrors are obtained at $1 \mathrm{k} \Omega, 6 \mathrm{k} \Omega, 10 \mathrm{nH}$, and $20 \mathrm{nH}$ values of impedance. The peaking in the frequency response depends upon the value of impedance used for compensation and this limits the value of impedance.

Figure 8 is the plot of output voltage requirement of modified LVCCM. In Figure 8, the output current gets saturated after $0.25 \mathrm{~V}$ and thus it can be concluded that the minimum output voltage requirement of the compensated LVCCM is $0.25 \mathrm{~V}$.

The frequency responses of conventional and wideband LVCCM are shown in Figure 9. The bandwidth is extended from $4.95 \mathrm{GHz}$ to $11.10 \mathrm{GHz}$ by using $R=6 \mathrm{k} \Omega$ and $18.94 \mathrm{GHz}$ by using $L=20 \mathrm{nH}$.

The input impedance of both conventional and compensated LVCCM is approximately $8 \Omega$. Table 3 summarizes the bandwidth of conventional and compensated LVCCM.

The circuit parameters of the proposed wideband high compliance current mirror are the same as taken in [19]. The input impedance of the proposed wideband high frequency current mirror is approximately $8 \Omega$ and does not change with the modification in the mirror.

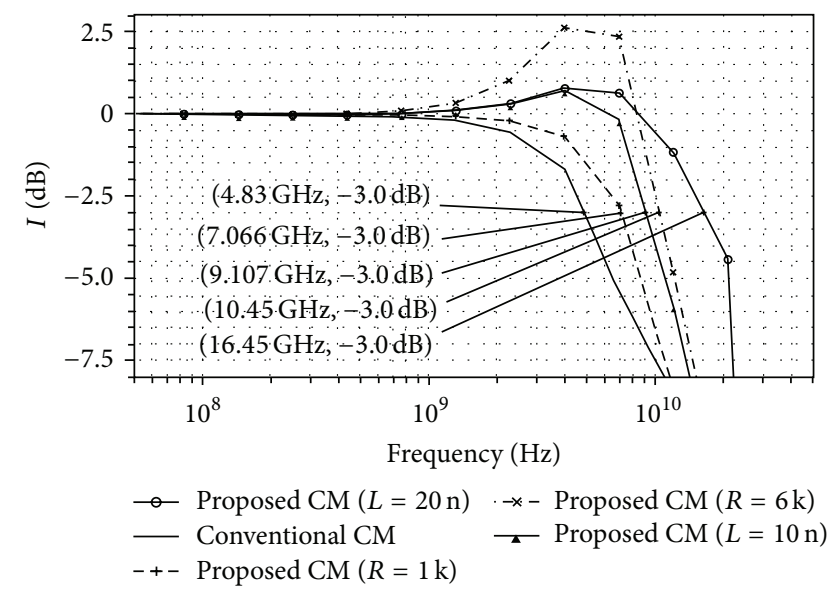

FIGURE 11: Frequency responses of the conventional and proposed current mirrors (where CM refers to current mirror).

The output current variation with output voltage is shown in Figure 10. The output compliance voltage is $66.368 \mathrm{mV}$. The output compliance voltages of the conventional and proposed current mirror are the same.

The DC power dissipation in the proposed current mirror is the same as that in the conventional one [19] and it is $21.611 \mu \mathrm{W}$. The frequency response of the proposed current mirror is shown in Figure 11 and it is compared with the conventional current mirror introduced in [19]. The BWER of the proposed current mirror is 3.40 and it is obtained by using $20 \mathrm{nH}$ inductance.

The Monte Carlo simulation of the proposed current mirror with resistively compensated LVCCM $(R=6 \mathrm{k} \Omega)$ results in mean deviation of $-496.63 e-12$ and standard deviation of $11.516 e-9$. The current mirror with inductively peaked LVCCM $(L=20 \mathrm{nH})$ has shown a mean deviation of $-532.47 e-12$ and a standard deviation of $11.941 e-9$ in the Monte Carlo simulation by using Pspice (OrCAD). The simulation results of the proposed current mirror are given in Table 4.

\section{Conclusion}

In this work, the bandwidth of a high compliance current mirror is extended without affecting its DC characteristics. The FVF based LVCCM is the basic building block of the proposed current mirror; therefore, in this paper we have modified LVCCM by using compensation techniques; namely, resistive compensation and inductive peaking. The BWER of high frequency LVCCM is 2.24 and 3.82 by using $6 \mathrm{k} \Omega$ resistance and $20 \mathrm{nH}$ inductance, respectively. The high frequency LVCCM is then used to replace the conventional one in the proposed current mirror. The BWER of the proposed wideband high compliance current mirror is 2.16 by using $6 \mathrm{k} \Omega$ resistance and 3.40 by using $20 \mathrm{nH}$ inductance, without affecting its linearity and input impedance. The analytical derivations and simulation results are justifying the approach used in this paper for bandwidth extension. The proposed wideband current mirror can be employed 
TABLE 3: Bandwidth comparison of conventional and proposed LVCCM.

\begin{tabular}{lcccccc}
\hline \multirow{2}{*}{ Performance factors } & & \multicolumn{2}{c}{ LVCCM } \\
& Conventional & $R=1 \mathrm{k} \Omega$ & $R=6 \mathrm{k} \Omega$ & $L=10 \mathrm{nH}$ & $L=20 \mathrm{nH}$ \\
\hline Bandwidth $(\mathrm{GHz})$ & 4.95 & 7.36 & 11.10 & 9.64 & 18.94 \\
\hline
\end{tabular}

TABLE 4: Bandwidth comparison of conventional and proposed current mirrors.

\begin{tabular}{lccccc}
\hline \multirow{2}{*}{ Performance factors } & \multicolumn{4}{c}{ High compliance current mirror } \\
& Conventional $[19]$ & $R=1 \mathrm{k} \Omega$ & $R=6 \mathrm{k} \Omega$ & $L=10 \mathrm{nH}$ & $L=20 \mathrm{nH}$ \\
\hline Bandwidth $(\mathrm{GHz})$ & 4.83 & 7.06 & 10.45 & 9.11 & 16.45 \\
\hline
\end{tabular}

in various analog integrated circuits for signal processing applications.

\section{Conflict of Interests}

The authors declare that there is no conflict of interests regarding the publication of this paper.

\section{References}

[1] S. S. Mohan, M. Del Mar Hershenson, S. P. Boyd, and T. H. Lee, "Bandwidth extension in CMOS with optimized on-chip inductors," IEEE Journal of Solid-State Circuits, vol. 35, no. 3, pp. 346-355, 2000.

[2] S. Galal and B. Razavi, "40-Gb/s amplifier and ESD protection circuit in 0.18- $\mu \mathrm{m}$ CMOS technology," IEEE Journal of SolidState Circuits, vol. 39, no. 12, pp. 2389-2396, 2004.

[3] B.-D. Liu, C.-Y. Huang, and H.-Y. Wu, "Modular current-mode defuzzification circuit for fuzzy logic controllers," Electronics Letters, vol. 30, no. 16, pp. 1287-1288, 1994.

[4] H. Hassan, M. Anis, and M. Elmasry, "MOS current mode circuits: analysis, design, and variability," IEEE Transactions on Very Large Scale Integration (VLSI) Systems, vol. 13, no. 8, pp. 885-898, 2005.

[5] M. H. Cohen and A. G. Andreou, "Current-mode subthreshold MOS implementation of the Herault-Jutten autoadaptive network," IEEE Journal of Solid-State Circuits, vol. 27, no. 5, pp. 714$727,1992$.

[6] C.-L. Chen, W.-L. Hsieh, W.-J. Lai, K.-H. Chen, and C.-S. Wang, "A high-speed and precise current sensing circuit with bulk control (CCB) technique," in Proceedings of the 15th IEEE International Conference on Electronics, Circuits and Systems (ICECS '08), pp. 283-287, September 2008.

[7] C. Wang and R. Zhao, "Continuous-time current-mode current mirror band pass filters with improved leap-frog structure," in Proceedings of the 1st International Congress on Image and Signal Processing (CISP '08), pp. 146-148, May 2008.

[8] G. Souliotis and C. Psychalinos, "Current-mode linear transformation filters using current mirrors," IEEE Transactions on Circuits and Systems II, vol. 55, no. 6, pp. 541-545, 2008.

[9] S. S. Rajput and S. S. Jamuar, "Low voltage, low power and high performance current conveyors for low voltage analog and mixed mode signal processing applications," Analog Integrated Circuits and Signal Processing, vol. 41, no. 1, pp. 21-34, 2004.
[10] E. Farshidi and H. Asiaban, "A new true RMS-to-DC converter using up-down translinear loop in CMOS technology," Analog Integrated Circuits and Signal Processing, vol. 70, no. 3, pp. 385390, 2012.

[11] E. Bruun and P. Shah, "Dynamic range of low-voltage cascode current mirrors," in Proceedings of the 1995 IEEE International Symposium on Circuits and Systems (ISCAS '95), pp. 1328-1331, May 1995.

[12] A. Garimella, L. Garimella, J. Ramirez-Angulo, A. J. LópezMartín, and R. G. Carvajal, "Low-voltage high performance compact all cascode CMOS current mirror," Electronics Letters, vol. 41, no. 25, pp. 1359-1360, 2005.

[13] F. Ledesma, R. Garcia, and J. Ramírez-Angulo, "Comparison of new and conventional low voltage current mirrors," in Proceedings of the 45th Midwest Symposium on Circuits and Systems, pp. II49-II52, August 2002.

[14] J. Ramírez-Angulo, R. G. Carvajal, and A. Torralba, "Low supply voltage high-performance CMOS current mirror with low input and output voltage requirements," IEEE Transactions on Circuits and Systems II, vol. 51, no. 3, pp. 124-129, 2004.

[15] R. G. Carvajal, J. Ramirez-Angulo, A. Lopez Martin, A. Torralba, J. Galan et al., "The flipped voltage follower: a useful cell for low voltage low power circuit design," IEEE Transactions on Circuits and Systems I, vol. 52, no. 7, pp. 1276-1279, 2005.

[16] L. Lee, R. M. Sidek, S. S. Jamuar, and S. Khatun, "Low voltage cascode current mirror in a $1.8 \mathrm{GHz}$ variable gain low noise amplifier (VGLNA)," in Proceedings of the International Symposium on Communications and Information Technologies (ISCIT '06), pp. 1097-1100, October 2006.

[17] H. Sato, A. Hyogo, and K. Sekine, "A low voltage OTA using MOSFET in the triode region and cascode current mirror," in Proceedings of the European Conference on Circuit Theory and Design, pp. III/453-III/456, September 2005.

[18] Y. Cong and R. L. Geiger, "Cascode current mirrors with low input, output and supply voltage requirements," in Proceedings of the Midwest Circuits and Systems Conference (MWSCAS '00), vol. 1, pp. 490-493, August 2000.

[19] S. Javad Azhari, H. Faraji Baghtash, and K. Monfaredi, "A novel ultra-high compliance, high output impedance low power very accurate high performance current mirror," Microelectronics Journal, vol. 42, no. 2, pp. 432-439, 2011.

[20] T. Voo and C. Toumazou, "High-speed current mirror resistive compensation technique," IEE Electronics Letters, vol. 31, no. 4, pp. 248-250, 1995.

[21] M. Gupta and U. Singh, "A new flipped voltage follower with enhanced bandwidth and low output impedance," Analog 
Integrated Circuits and Signal Processing, vol. 72, no. 1, pp. 279288, 2012.

[22] S. Shekhar, J. S. Walling, and D. J. Allstot, "Bandwidth extension techniques for CMOS amplifiers," IEEE Journal of Solid-State Circuits, vol. 41, no. 11, pp. 2424-2438, 2006.

[23] U. Singh and M. Gupta, "High frequency flipped voltage follower with improved performance and its application," Microelectronics Journal, vol. 44, no. 12, pp. 1175-1192, 2013.

[24] D. J. Comer, D. T. Comer, J. B. Perkins, K. D. Clark, and A. P. C. Genz, "Bandwidth extension of high-gain CMOS stages using active negative capacitance," in Proceedings of the 13th IEEE International Conference on Electronics, Circuits and Systems (ICECS '06), pp. 628-631, December 2006.

[25] W. Sansen and Z. Y. Chang, "Feedforward compensation techniques for high-frequency CMOS amplifiers," IEEE Journal of Solid-State Circuits, vol. 25, no. 6, pp. 1590-1595, 1990.

[26] M. Gupta, P. Aggarwal, P. Singh, and N. K. Jindal, "Low voltage current mirrors with enhanced bandwidth," Analog Integrated Circuits and Signal Processing, vol. 59, no. 1, pp. 97-103, 2009.

[27] M. Ebrahimzadeh, "A low voltage, high quality factor floating gate tunable active inductor with independent inductance and quality factor tuning," International Journal of Computer and Electrical Engineering, vol. 3, no. 2, pp. 180-183, 2011.

[28] S. Adel Sedra and C. Kenneth Smith, Microelectronics Circuits, Oxford University Press, New York, NY, USA, 5th edition, 2005. 

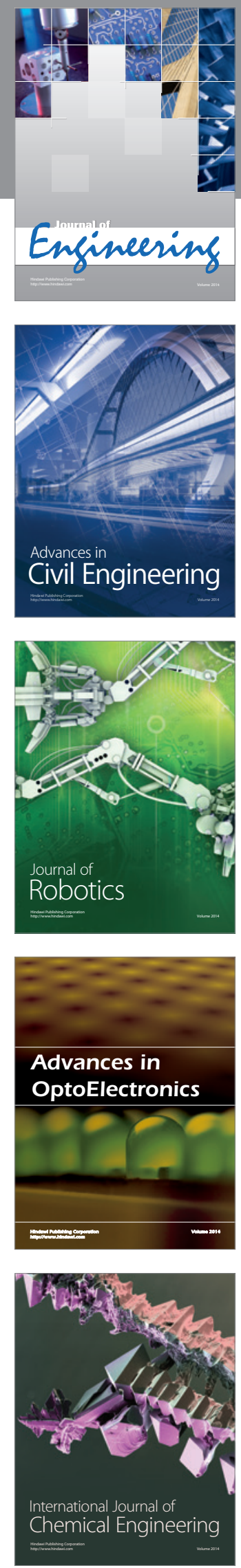

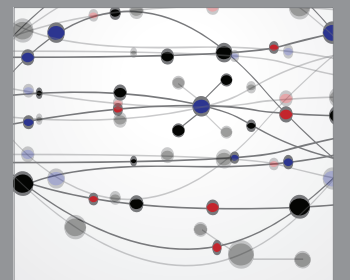

The Scientific World Journal
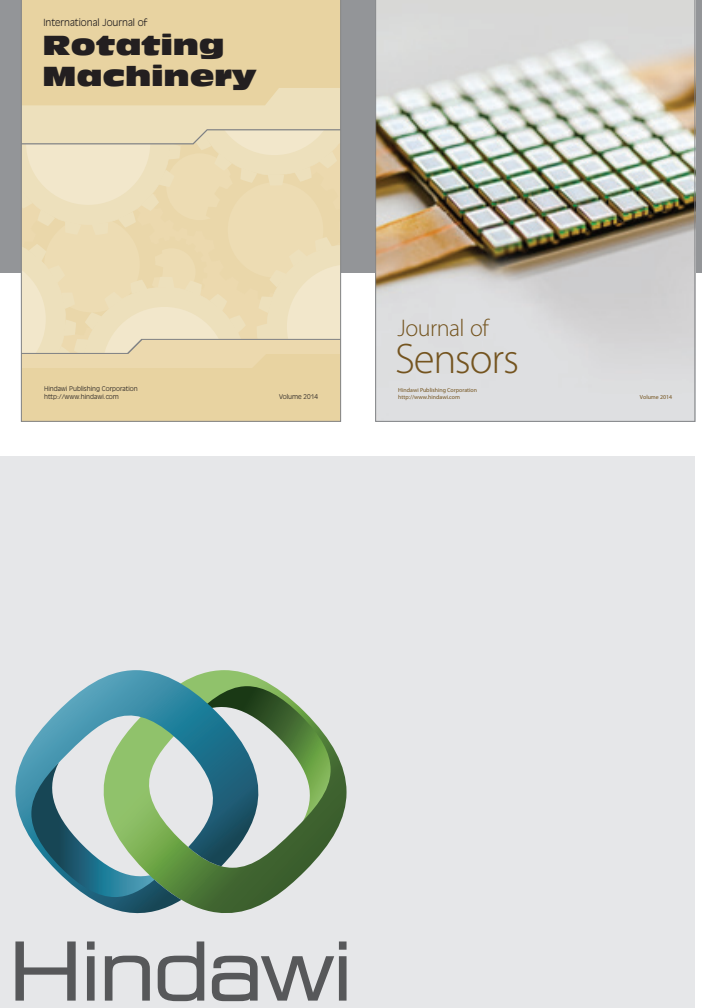

Submit your manuscripts at http://www.hindawi.com
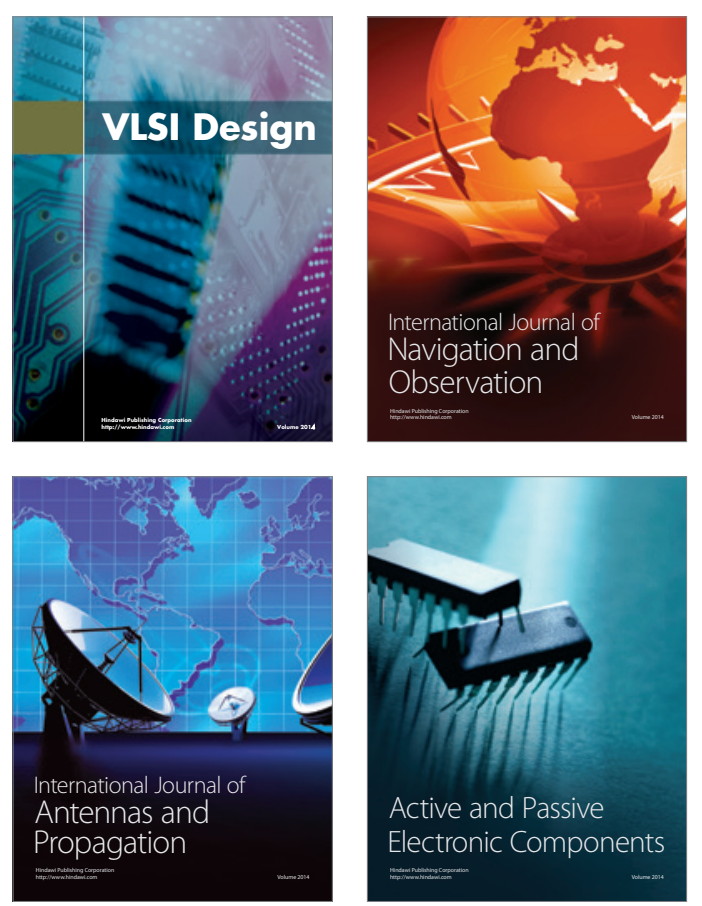
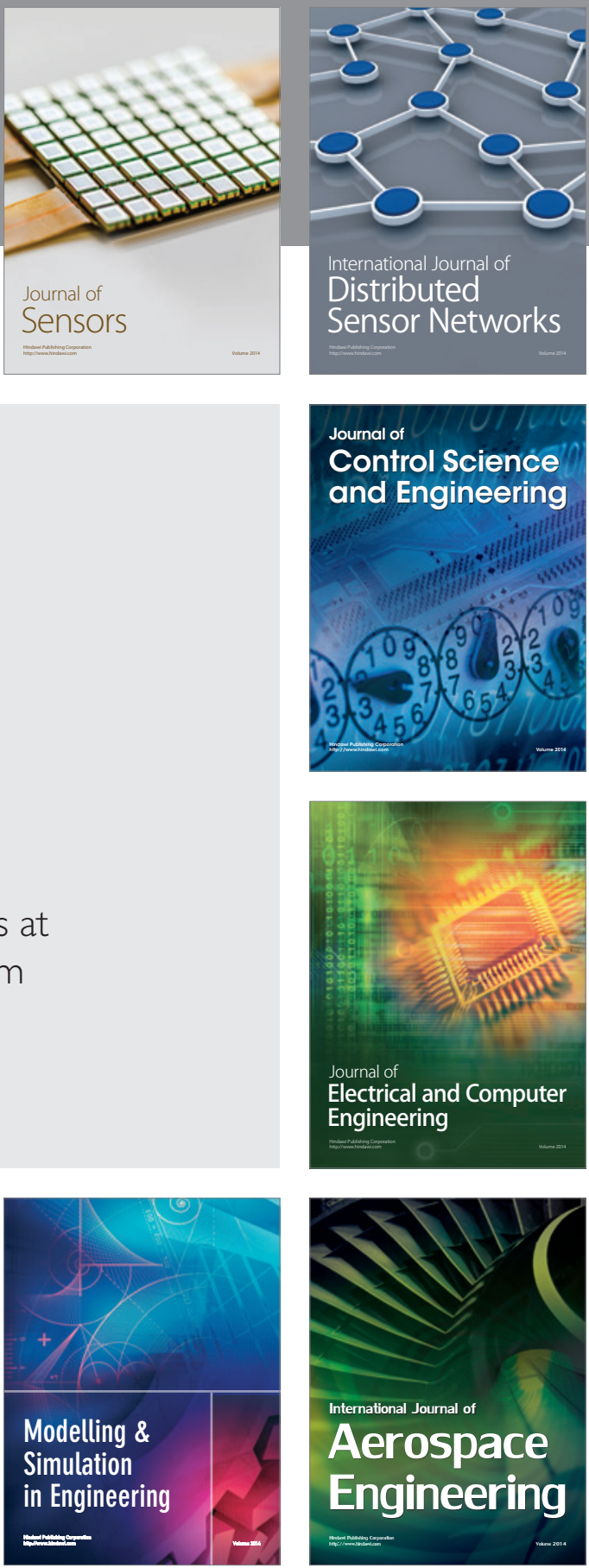

Journal of

Control Science

and Engineering
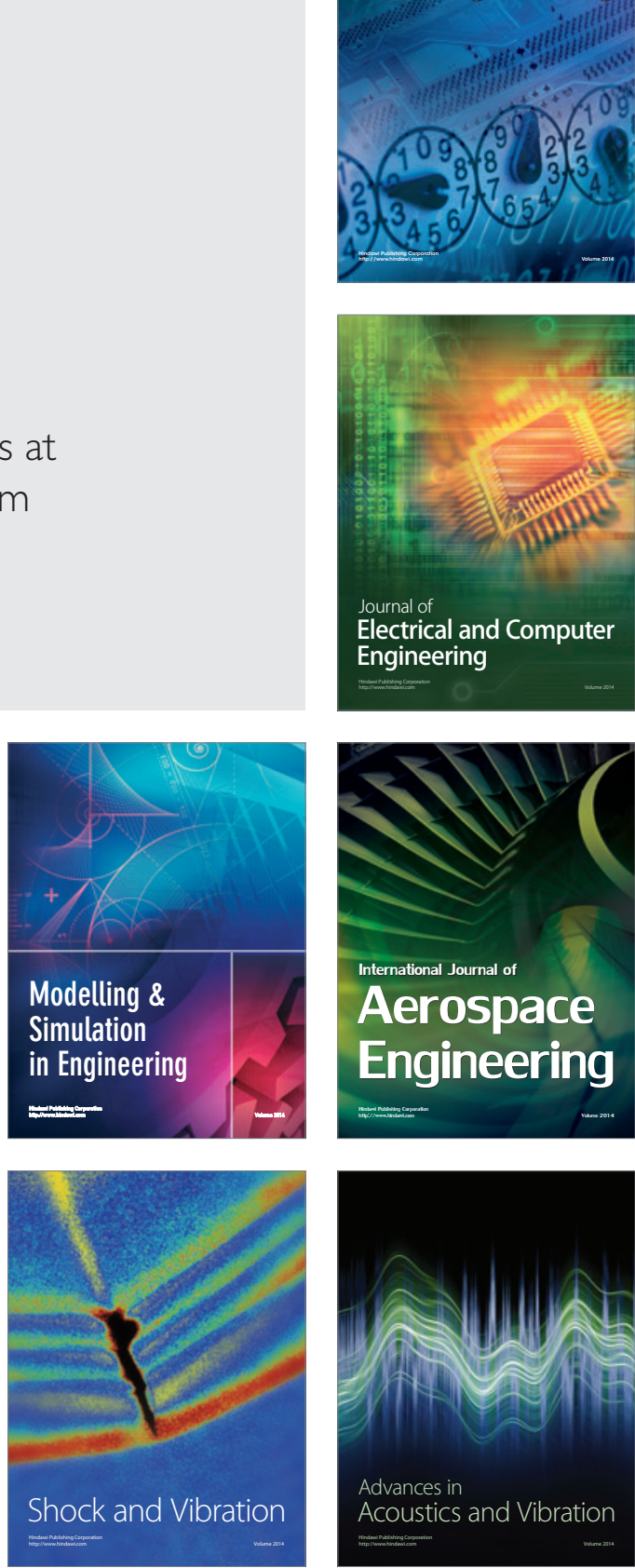